PROCEEDINGS OF THE

AMERICAN MATHEMATICAL SOCIETY

Volume 130, Number 4, Pages 1207-1210

S 0002-9939(01)06448-6

Article electronically published on November 9, 2001

\title{
A BERNSTEIN TYPE THEOREM FOR MINIMAL VOLUME PRESERVING MAPS
}

LEI NI

(Communicated by Bennett Chow)

\begin{abstract}
We show that any minimal volume preserving map from the Euclidean plane into itself is a linear diffeomorphism. We derive this from a similar result on minimal diffeomorphisms. We also show that the classical Bernstein theorem on minimal graphs is a corollary of our result.
\end{abstract}

\section{IntRoduction}

In [2], Bernstein proved that the entire, two-dimensional, minimal graph must be a hyperplane. Its generalization to higher dimensions has been the core problem in the study of the minimal submanifolds and the nonlinear elliptic PDE. One can refer to [1], [9], 3], etc., for the history of the development. It was also generalized by studying the image of the Guass maps in [7, 11] and [5]. In [4, it was further generalized to the complete stable minimal hypersurface in $R^{3}$. Very recently, it was generalized to affine maximum surfaces in [10. Here we are going to study the generalization to higher codimension. In particular, we will prove the following Bernstein type result:

Theorem 1. Any minimal volume preserving map from the Euclidean plane $R^{2}$ into $R^{2}$ is a linear diffeomorphism.

In general, a map between two Riemannian manifolds $M_{1}$ and $M_{2}$ is called minimal if the graph of the map is a minimal submanifold of the product $M_{1} \times M_{2}$. For example, Bernstein's theorem can be rephrased to say that any minimal maps from the Euclidean plane $R^{2}$ into a Euclidean line $R^{1}$ must be linear maps. We first show a result on minimal diffeomorphisms between the Euclidean plane $R^{2}$ 's. Then we derive Theorem 1 out of it by using results of Wolfson on minimal lagrangians. Our proof also shows that the classical Bernstein's theorem on the entire minimal graphs follows from our results. Thus the results we show are generalizations of the above-mentioned Bernstein's theorem.

\section{Minimal maps Between $R^{2}$ 's}

Let us start with the following result, which is essentially due to R. Schoen.

Received by the editors August 18, 2000.

2000 Mathematics Subject Classification. Primary 58E20.

Key words and phrases. Minimal maps, volume preserving, lagrangian submanifolds.

This research was partially supported by an NSF grant.

(C)2001 American Mathematical Society 
Theorem 2.1. A minimal diffeomorphism from the Euclidean plane $R^{2}$ into $R^{2}$ is a linear diffeomorphism.

Proof. The proof is based on two facts. The first comes from the minimal surface theory. Let $f: R^{2} \rightarrow R^{2}$ be a minimal diffeomorphism. In coordinates, one can write it as

$$
f:(x, y) \rightarrow(u(x, y), v(x, y)) .
$$

By the assumption we know that

$$
\sum=\left\{(x, y, u(x, y), v(x, y)) \in R^{4} \mid(x, y) \in R^{2}\right\}
$$

is a minimal surface. By the minimal surface theory we know that through the so-called Lewy's map there exists a coordinates change in the form of

$$
\begin{aligned}
& x_{1}=x, \\
& x_{2}=a x+b y
\end{aligned}
$$

such that $\left(x_{1}, x_{2}\right)$ are the isothermal parameters for $\sum$ (cf. Theorem 5.1 of [7]). Then we know that $u\left(x_{1}, x_{2}\right)$ and $v\left(x_{1}, x_{2}\right)$ are two harmonic functions in terms of $\left(x_{1}, x_{2}\right)$. Thus the map $f$, viewed in terms of the new coordinates system on the domain, is a harmonic map, a harmonic diffeomorphism in this case. The theorem then follows from the second well-known fact from the harmonic maps theory (cf. [8]). Namely, the only harmonic diffeomorphisms between the complex planes are linear diffeomorphisms. For the sake of completeness we also include a proof here. First let $z=x_{1}+\sqrt{-1} x_{2}$ and $f=u+\sqrt{-1} v$. $f$ being harmonic means $f_{z \bar{z}}=0$. Now assume that $f$ is orientation preserving. Thus $\left|f_{z}\right|>\left|f_{\bar{z}}\right|$. In particular, $f_{z} \neq 0$. Let $g=\frac{\overline{f_{z}}}{f_{z}}$. The harmonicity of $f$ implies that $g$ is a holomorphic function and $|g|<1$. Therefore $g$ is a constant with module less than 1 . Let us denote $g=A$. Then $f-\bar{A} \bar{f}$ is a holomorphic function. Now

$$
h:\left(x_{1}, x_{2}\right) \rightarrow f-\bar{A} \bar{f} \in \mathbf{C}
$$

is a holomorphic diffeomorphism, therefore a linear map. This implies that the original $f$ is a linear map from $\left(x_{1}, x_{2}\right)$ to $(u, v)$. Therefore the one from $(x, y)$ to $(u, v)$ is also a linear one.

Now we begin to prove Theorem 1 using Theorem 2.1. A priori, we do not know if a volume preserving minimal map is a diffeomorphism or not and it is suffcient to show that it is a diffeomorphism. In fact we can show that if $f$ : $(x, y) \rightarrow(u(x, y), v(x, y))$ is a volume preserving map, then it is a gradient map of a convex function $\psi(x, y)$ defined over $R^{2}$. In particular, $f$ is a diffeomorphism. The fact of being able to express a minimal volume preserving map as a gradient map was first derived for volume preserving minimal maps between simply-connected domains in [12]. We include the proof here for the sake of completeness. To prove that the map is a gradient map we need a general result of Wolfson on minimal lagrangians in Kähler-Einstein surfaces. Before we state the result, let us first introduce some notation. Let $X$ be a Kähler-Einstein manifold with the Kähler form $\omega$. In particular, $X$ is a symplectic manifold. A submanifold $L$ is called a Lagrangian submanifold if $\left.\omega\right|_{L}$ is identically zero. Let $K_{X}$ be the canonical line bundle of $X$. Then it was shown in [12] that $\left.K_{X}\right|_{L}$ is trivial and curvature of the induced hermitian metric is zero. Let $\sigma$ denote a section of $K_{X}$. Namely, $\sigma$ is an $(n, 0)$ form on $X$. For $p_{0} \in L$, pick a section $\sigma$ of $\left.K_{X}\right|_{L}$ at $p$ and then parallel translate it to obtain a global section $\sigma$ on $L$, in the case when $L$ is simply 
connected. Let $T_{p} L$ be the tangent bundle of $L$ at $p$. One can then define the so-called lagrangian angle $\beta$ of $L$ as follows:

$$
\frac{\sigma(x)\left(T_{x} L\right)}{|\sigma|(x)}=e^{\pi i \beta(x)} .
$$

It was shown that $\beta$ is well defined in $\mathbf{R} / 2 \mathbf{Z}$. A result of Wolfson says that

Theorem 2.2 (Corollary 1.5, [12]). Suppose that $X$ is a Kähler-Einstein manifold and $L$ is a lagrangian submanifold. If $L$ is also minimal, then $\beta$ is a constant function on each component of $L$.

Now we can apply the above result to our situation. Let $f$ be the volume preserving minimal map as in Theorem 1. Let $\omega_{1}=d x \wedge d y$ and $\omega_{2}=d u \wedge d v$ be the two symplectic forms on the domain and target $R^{2}$ 's. It is well known that being volume preserving is equivalent to the fact that $\sum$ defined as in the proof of Theorem 2.1 is a lagrangian submanifold in $R^{4}$ with respect to the symplectic form $\omega_{1}-\omega_{2}$, which can be thought as the Kähler form of $\mathbf{C} \times \overline{\mathbf{C}}$. Now we can have the a well-defined parallel holomorphic $(2,0)$ form $\Omega$,

$$
\Omega=(d x+\sqrt{-1} d y) \wedge(d u-\sqrt{-1} d v),
$$

on $\mathbf{C} \times \overline{\mathbf{C}}$. We use this to calculate the lagrangian angle of $\sum$. Direct calculation then shows that

$$
\frac{-\left(u_{x}+v_{y}\right)}{u_{y}-v_{x}}=\tan \pi \beta
$$

By the rotation of the target we can assume without loss of generality that $\beta=\frac{\pi}{2}$. Then we have that

$$
u_{y}=v_{x} .
$$

Therefore we know that there exists a function $\psi=\psi(x, y)$ such that

$$
u=\psi_{x} \text { and } v=\psi_{y} .
$$

On the other hand, the volume preserving assumption implies that

$$
\psi_{x x} \psi_{y y}-\psi_{x y}^{2}=1 .
$$

From this, without loss of generality we can also assume that

$$
\psi_{x x}+\psi_{y y}>0 .
$$

Therefore $\psi$ is a convex function defined on $R^{2}$ and $f=\nabla \psi$. Now it is easy to see that $f$ is a diffeomorphism. Applying Theorem 2.1 gives the proof of Theorem 1.

As a corollary we have the following theorem of Jögens.

Corollary 2.3. The entire convex soltuion of the Monge-Ampère equation

$$
\operatorname{det}\left(D^{2} \psi\right)=1
$$

is a quadratic polynomial.

Proof. Let $f=\nabla \phi$. Then by assumption we know that $\psi$ is a volume preserving map. Let $\sum$ be the graph of $f$. Then it is a lagrangian in $R^{2}$. Now we reverse the calculation in the proof of Theorem 1 and conclude that $\beta \equiv 0$. Then Theorem 1.2 or Theorem 1.3 of [12] implies that $\sum$ is also minimal. The result follows from Theorem 2.1.

It was shown in [6] that the classical Bernstein's theorem is an easy consequence of the above result of Jögens, and is therefore a consequence of Theorem 1 or Theorem 2.1. 


\section{ACKNOWLEDGEMENT}

The author would like to thank Tom Wan for pointing out that Theorem 2.1 was proved earlier by R. Schoen and Jon Wolfson for various discussions.

\section{REFERENCES}

[1] F. Almgren, Some interior regularity theorems for minimal surfaces and an extension of Bernstein's theorem, Ann. Math. 74(1966), 277-292. MR 34:702

[2] S. Bernstein, Sur un theoreme de geometrie et ses applications aux equations aux derivees partielle du type elliptique, Comm. de la Soc. Math de Kharkov 15(1915-17), 38-45.

[3] E. Bombieri, E. De Giorgi, E. Giusti, Minimal cones and the Bernstein problem, Invent. Math. 7(1969), 243-268. MR 40:3445

[4] D. Fischer-Colbrie, R. Schoen, The structure of complete stable minimal surfaces in 3manifolds of nonnegative scalar curvature, Comm. Pure Appl. Math. 33(1980), no. 2, 199211. MR 81i:53044

[5] H. Fujimoto, Modified defect relations for the Gauss map of minimal surfaces, J. Differential Geom. 29(1989), no. 2, 245-262. MR 89m:53012

[6] J. Nitsche, Elementary proof of Bernstein's theorem on minimal surfaces, Ann. of Math. (2) 66(1957), 543-544. MR 19:878f

[7] R. Osserman, A survey of minimal surfaces, Van Nostrand-Reinhold, New York, 1969. MR 41:934

[8] R. Schoen, The role of harmonic mappings in rigidity and deformation problems, Complex geometry (Osaka, 1990), 179-200, Lecture Notes in Pure and Appl. Math., 143, Dekker, New York, 1993. MR 94g:58055

[9] J. Simons, Minimal varieties in riemannian manifolds, Ann. of Math. (2) 88(1968), 62-105. MR 38:1617

[10] N. Trudinger, X. Wang, The Bernstein problem for affine maximal hypersurfaces Invent. Math. 140(2000), no. 2, 399-422. MR 2001h:53016

[11] F. Xavier, The Gauss map of a complete nonflat minimal surface cannot omit 7 points of the sphere, Ann. of Math. (2) 113(1981), no. 1, 211-214. MR 82b:53015 Erratum MR 83h:53016

[12] J. Wolfson, Minimal Lagrangian diffeomorphisms and the Monge-Ampère equation, J. Differential Geom. 46 (1997), no. 2, 335-373. MR 99e:58045

Department of Mathematics, Stanford University, Stanford, California 94305

E-mail address: Ini@math.stanford.edu 